\title{
Successful Stabilization of Symptomatic Bone Marrow Metastasis Two Times in a Breast Cancer Patient
}

\author{
HIDEKO AKAGI ${ }^{1}$, AKIHIKO SHIMADA $^{2}$, KENKO CHIN $^{3}$ and HIDEHARU DOMOTO ${ }^{4}$ \\ Departments of ${ }^{1}$ Oncology, ${ }^{2}$ Surgery, ${ }^{3}$ Hematology, and ${ }^{4}$ Pathology, Keiyu Hospital, Yokohama, Japan
}

\begin{abstract}
Background: Bone marrow metastasis is very uncommon in breast cancer. Cancer patients showing a dramatic response to chemotherapy with full recovery are very rare. Case Report: This is a case report of a 62-yearold woman who underwent partial mastectomy six years previously. The patient presented with increased fatigue and her hemoglobin level was $6.7 \mathrm{~g} / \mathrm{dl}$. Pathological examination of a bone marrow biopsy showed metastasis from breast cancer. Systemic therapy was initiated with doxorubicin and cyclophosphamide and pancytopenia was steadily improved. However, 15 months later, she felt severe fatigue again. Eribulin was administered and the patient showed sufficient recovery. She had two bone marrow metastases that caused pancytopenia including severe anemia. However, she survived twice with chemotherapy. Conclusion: Bone marrow metastasis of breast cancer is life-threatening; however, chemotherapy may significantly improve survival.
\end{abstract}

Breast cancer is the most common malignancy in women worldwide (1). Although metastatic disease, considered incurable, is rarely seen at the time of the initial diagnosis, approximately $20 \%$ of women with operable breast cancer eventually relapse, with approximately $70 \%$ of relapses occurring as distant metastasis (2-5). Although bone marrow metastasis is commonly present among breast cancer patients, total bone marrow metastasis resulting in profound pancytopenia is extremely rare $(6,7)$. Symptomatic bone marrow metastasis is an uncommon presentation of metastatic breast cancer. A retrospective study by Kopp et al. showed that out of 12,970 patients with bone marrow

This article is freely accessible online.

Correspondence to: Hideko Akagi, MD, Ph.D., Department of Oncology, Keiyu Hospital, Yokohama, Kanagawa 220-8521, Japan. Tel: +81 (45)2218181, e-mail: h-akagi@keiyu-hospital.com

Key Words: Pancytopenia, metastatic breast cancer, bone marrow metastasis, doxorubicin, cyclophosphamide, eribulin. metastasis only $0.17 \%$ had symptomatic bone marrow metastasis. The management of patients with bone metastasis also creates confusion regarding treatment decisions. Clinicians need to approach cytotoxic treatment carefully, since these patients have marrow failure (8).

\section{Case Report}

The patient was a 62-year-old woman who had been diagnosed six years previously with poorly-differentiated invasive lobular carcinoma that was positive for estrogen receptor (ER), but negative for progesterone receptor $(\mathrm{PgR})$ and HER2/neu. Modified partial mastectomy with axillary lymph node dissection was then performed. The stage at the time was T2N0M0. The patient subsequently received an aromatase inhibitor, (anastrozole) as adjuvant hormone treatment. After three years of treatment, bone metastasis was detected. She then underwent palliative bone radiation and was treated with denosumab, fulvestrant and toremifene for two years. As her tumor marker levels increased, the treatment was changed to tremifen. After one year of treatment with tremifen, multiple hepatic metastases were detected. She presented with increased fatigue that was interfering with her daily living activities. On a physical examination, she looked pale and fatigued. Her palpebral conjunctiva suggested anemia. Her heart rate was 114 beats/min. The bilateral lungs were clear and a cardiovascular exam was normal, with no murmurs. Her abdomen was soft and nontender without organomegaly. Her breasts were unchanged including her left breast scars.

Peripheral blood laboratory tests indicated pancytopenia. Her white blood cell (WBC) count was $4,100 / \mu 1$, her hemoglobin (Hgb) level was $6.8 \mathrm{~g} / \mathrm{dl}$, and her platelet count was $92,000 / \mu \mathrm{l}$. Her lactate dehydrogenase (LDH) was slightly elevated at 254 U/1. Her carcinoembryonic antigen (CEA) level was $27.8 \mathrm{ng} / \mathrm{ml}$ and her carbohydrate antigen 15-3 level was $70.5 \mathrm{U} / \mathrm{ml}$. These levels were higher than those at the previous examination. Abdominal magnetic resonance imaging (MRI) revealed multiple hepatic metastases. Bone marrow aspiration and biopsy was also performed. Examination of the bone marrow biopsy 
revealed the formation of glandular cavities and metastatic carcinoma infiltrating in an alveolar shape (Figure 1A). Immunohistochemistry revealed that the bone marrow specimen was positive for estrogen receptor and pancytokeratin (AE1/AE3) (Figure 1B and 1C). These findings were consistent with bone marrow metastasis from breast cancer (Figure 1).

After a thorough discussion about the risks and benefits of her therapy in view of her profound pancytopenia, systemic therapy was initiated with doxorubicin $\left(90 \mathrm{mg} / \mathrm{m}^{2}\right)$ and cyclophosphamide $\left(600 \mathrm{mg} / \mathrm{m}^{2}\right)$ every three weeks. Both doses were reduced by $20 \%$ from the first cycle because of her pancytopenia. In addition to doxorubicin and cyclophosphamide, she received zoledronic acid as a part of the management for osseous metastasis from breast cancer. Doxorubicin and cyclophosphamide were transitioned to triweekly docetaxel at the end of the eighth doxorubicin and cyclophosphamide treatment. The docetaxel dose was 75 $\mathrm{mg} / \mathrm{m}^{2}$ and was reduced by $20 \%$ from the first cycle of docetaxel. During chemotherapy, she tolerated treatment moderately, with several red blood cell transfusions, platelet transfusions, and injections of G-CSF. As shown in Figure 2, the patient's pancytopenia steadily improved after the initiation of chemotherapy. At approximately seven months after the start of treatment, she experienced a near full recovery following several red blood and platelet transfusions. Her pancytopenia steadily improved after the initiation EC. Her laboratory tests revealed a WBC count of $4,000 / \mu \mathrm{l}$, an $\mathrm{Hgb}$ level of $8.5 \mathrm{~g} / \mathrm{dl}$ and platelet counts of $95,000 / \mu \mathrm{l}$ at that time, indicating a remarkable response to chemotherapy. WBC, which was previously reduced by anticancer drug treatment, was almost normalized without GCSF. At approximately, nine months after commencing treatment, her WBC count increased to $3,900 / \mu \mathrm{l}, \mathrm{Hgb}$ increased to $9.0 \mathrm{~g} / \mathrm{dl}$ and her platelet count improved to $149,000 / \mu 1$. Her tumor markers levels, including CEA and CA15-3, were decreasing during chemotherapy. At nine months after treatment, her CEA level decreased from 27.8 $\mathrm{ng} / \mathrm{ml}$ to $6.1 \mathrm{ng} / \mathrm{ml}$. Her CA15-3 level decreased from 70.5 $\mathrm{U} / \mathrm{ml}$ to $41.4 \mathrm{U} / \mathrm{ml}$ (Figure 2). After three cycles of docetaxel, MRI demonstrated that her multiple liver metastases had shrunk. Bone marrow aspiration and biopsy was performed at approximately the same time. A pathological examination of the bone marrow biopsy specimen revealed that the recurrence of breast cancer had disappeared when the patient's pancytopenia disappeared.

After eight cycles of docetaxel, the patient was transitioned to hormone treatment, with exemestane because numbness, which was an adverse effect of docetaxel, caused inconvenience in her life. However, at approximately six months after the start of hormone treatment, MRI detected multiple liver metastases. The therapy was switched back to docetaxel. After two cycles of docetaxel, she had survived fifteen months without transfusion. Unfortunately, after four cycles of docetaxel, she felt severe fatigue and had a high fever of almost $38.5^{\circ} \mathrm{C}$ every day. Her hemoglobin (Hgb) level was $5.3 \mathrm{~g} / \mathrm{dl}$, and her platelet count was 5,900/ $\mu 1$. A second relapse was suspected. At that time, her CEA level increased to $205 \mathrm{ng} / \mathrm{ml}$ and CA15-3 increased to $284 \mathrm{U} / \mathrm{ml}$. Her pancytopenia was so severe that we thought she would lose her life if the new treatment was not succeeded. We therefore decided to administer eribulin in the next treatment line, with several red blood cell transfusions, platelet transfusions and G-CSF infusions. Her state of pancytopenia remained for one and a half months, even though eribulin was only administered once. However, she experienced a near full recovery from her pancytopenia for almost two months. At that time, her hemoglobin (Hgb) level was $9.5 \mathrm{~g} / \mathrm{dl}$, and her platelet counts were $8600 / \mu \mathrm{l}$. Her CEA level decreased to $102.6 \mathrm{ng} / \mathrm{ml}$ and her CA15-3 level decreased to $123 \mathrm{U} / \mathrm{ml}$ (Figure 3). After recovering from pancytopenia, it was desirable to continue eribulin. She wanted to change the oral anticancer drug because of her numbness from long-lasting chemotherapy. She was treated with tegaful/gimeracil/oteracil. This was continued for two weeks, with a one-week rest every three weeks. Her tumor marker levels increased steadily despite the administration of oral chemotherapy; there were no findings of progressive disease. However, at 14 months after the administration of oral chemotherapy, pain around the scapula suddenly appeared and she started to become conscious of stagger. Head MRI showed cancerous meningitis. Because she quickly began to experience disorientation, we discussed the treatment strategy with her and her husband. Eventually, she gave up chemotherapy and chose best supportive care.

Her laboratory profile remained stable for 21 months after the initiation of doxorubicin and cyclophosphamide at the first recurrence of bone marrow metastasis. Furthermore, she managed to maintain a normal life for 15 months after the second recurrence, following the initiation of eribulin. In her last two months of life, her disease progressed even in the meninges. However, pancytopenia was not seen until just before her death. She ultimately died from cancerous meningitis at 38 months after the diagnosis of bone marrow metastasis. The patient succeeded in overcoming symptomatic bone marrow metastasis of breast cancer twice.

\section{Discussion}

Cancer cell metastasis to the bone marrow is mainly asymptomatic. Cancer cells that infiltrate the bone marrow result in disturbed hematopoiesis, leading to cytopenia. Due to this loss of the normal function, hematological disorders, such as anemia, thrombocytopenia and leukoerythroblastosis, become clinically apparent (9). In some cases, tumor cell infiltration leads to total marrow failure or pancytopenia. In the present case, bone marrow was suspected based on a peripheral blood analysis, which showed pancytopenia. A 


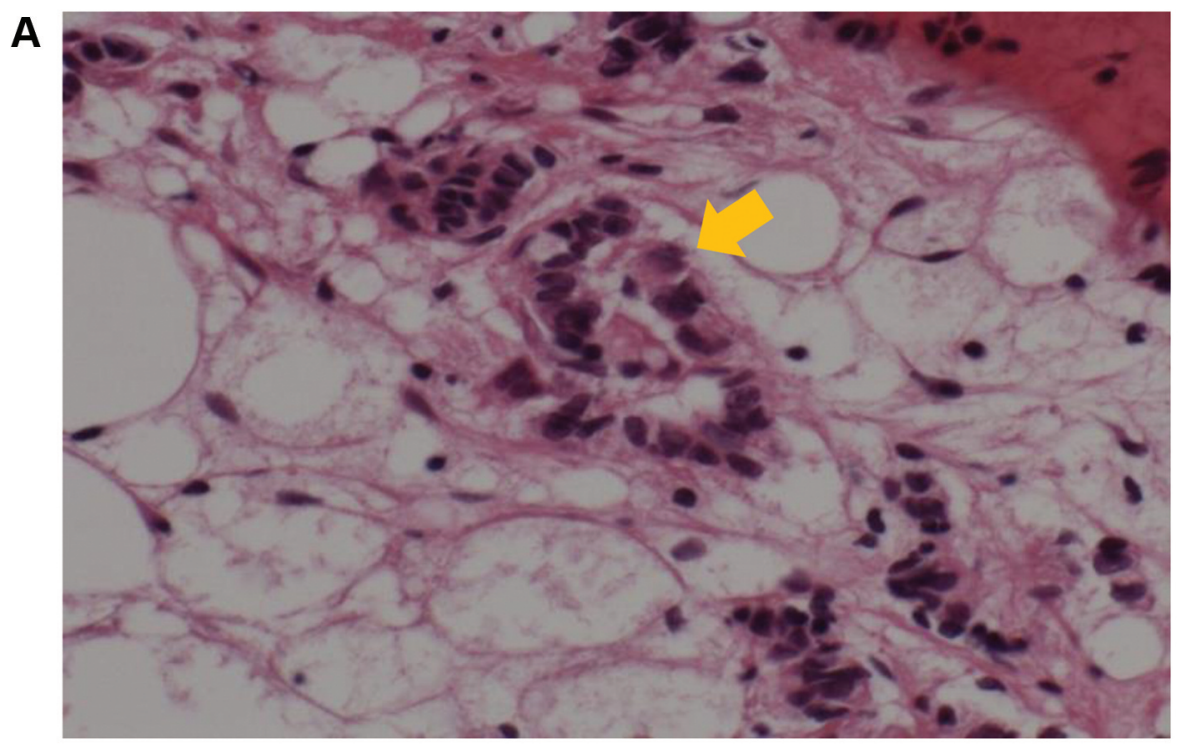

B

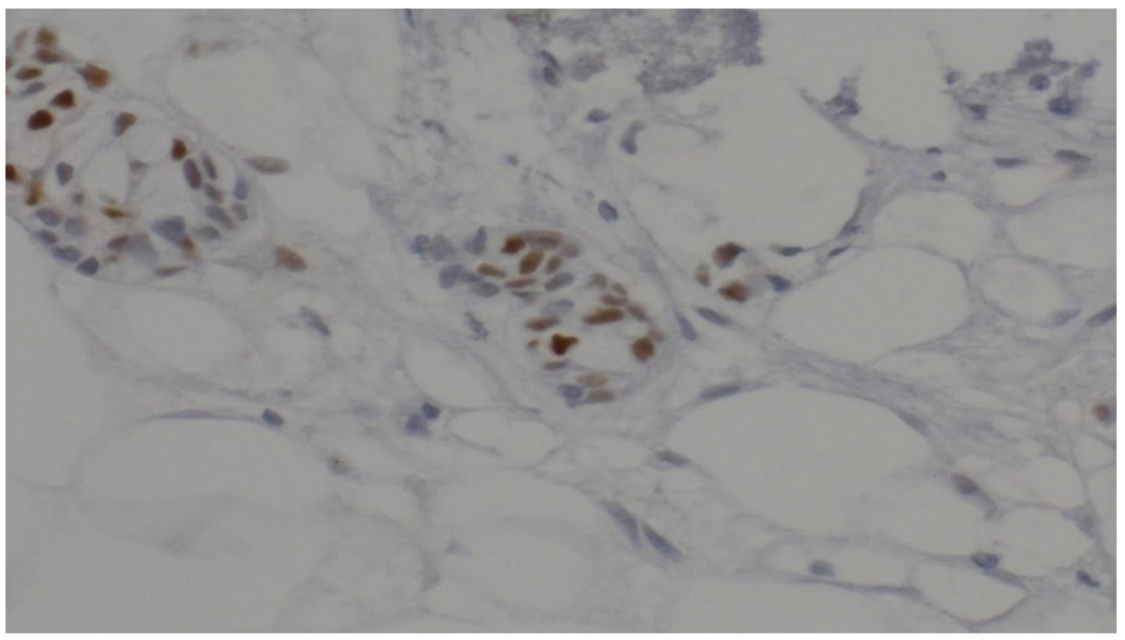

C

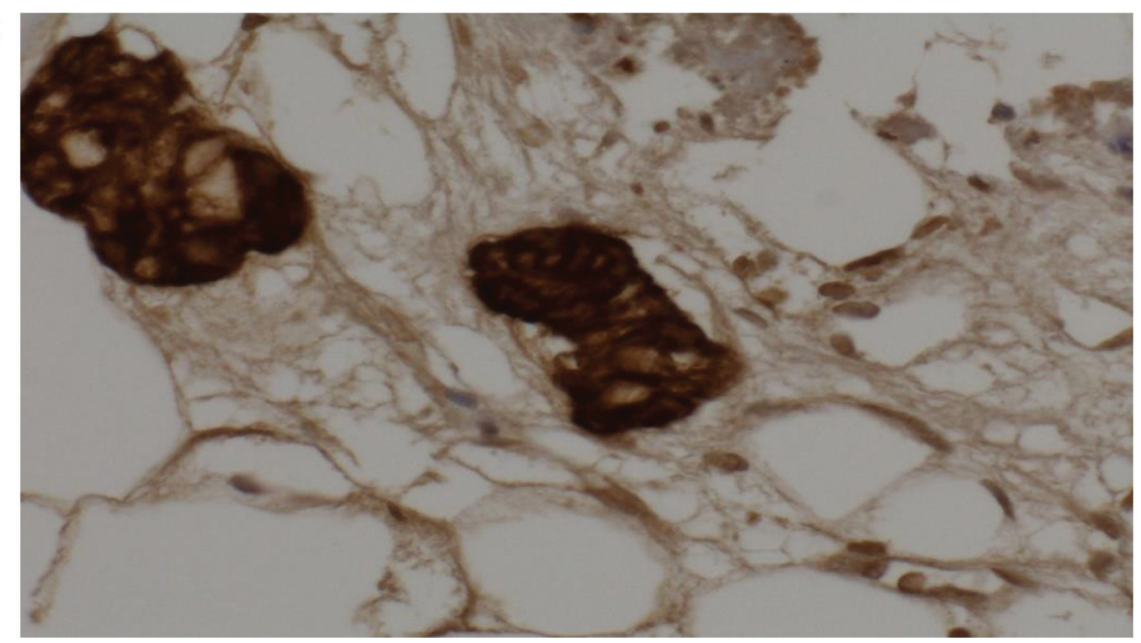

Figure 1. Pathological findings leading to a diagnosis of bone marrow metastasis. A. Bone marrow biopsy revealed the formation of glandular cavities with the infiltration of metastatic carcinoma in an alveolar shape (HE staining $\times 400$, arrow). B. Pathological analysis showed that the bone marrow was positive for estrogen receptors (HE staining $\times 400)$. C. A pathological analysis indicated that the bone marrow was positive for pancytokeratin (AE1/AE3) (HE staining $\times 400)$. 


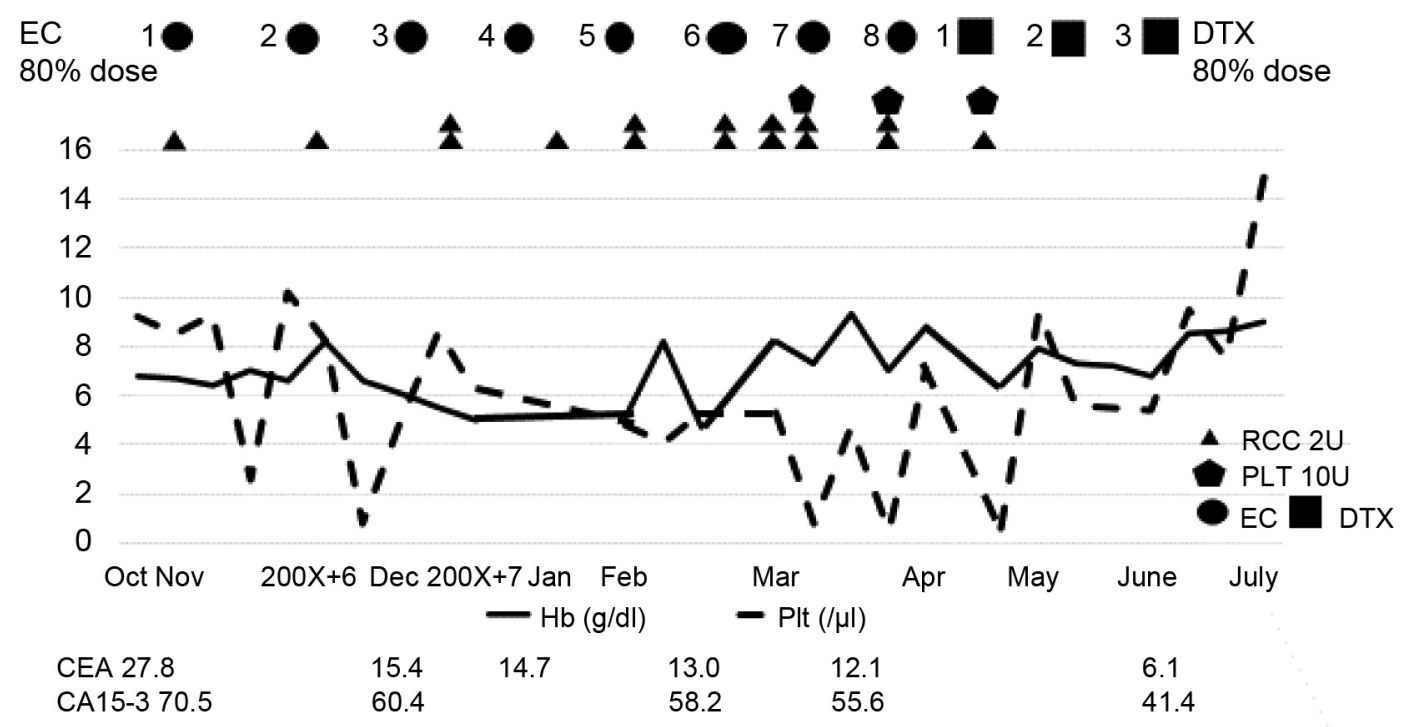

Figure 2. During chemotherapy treatment with doxorubicin and cyclophosphamide, both Hb levels and platelet counts were unstable due to the chemotherapy-induced myelosuppression, and tumor marker levels tended to decrease. Though blood transfusion was required until the first docetaxel treatment, blood transfusions have no longer been required since then. Hb levels and platelet counts stabilized. She survived the bone marrow metastasis of breast cancer for the first time.

peripheral blood smear revealed leukoerythroblastosis; cancer cell infiltration of the bone marrow was suspected (8). Therefore, a bone marrow biopsy was performed to confirm the diagnosis. In this case, a pathological examination of the bone marrow revealed estrogen receptor and pancytokeratin (AE1/AE3) positivity which was consistent with bone marrow metastasis (Figure 1).

A systemic search of the relevant English literature in Pubmed, including abstracts, retrieved a small number of reports (10-16) and one case (8). The most common histology of bone marrow metastasis resulting from breast cancer is invasive ductal carcinoma, followed by invasive lobular carcinoma. Hormone receptors are detected in 50$80 \%$ of cases $(8,13)$. The present case was characterized by invasive ductal carcinoma and hormone receptor positivity and thus shared similarities with previous reports.

Very limited data exist as to the safest and most effective way to treat patients with profound pancytopenia due to metastatic solid tumor involvement. The treatment of a patient with total marrow failure requires a cautious approach. In the limited studies supporting the optimal management of such cases, different results were obtained. Sasada et al. reported that a patient with total marrow failure caused by breast cancer metastasis died due to complications of pancytopenia that were likely caused by disseminated intravascular coagulation and metastatic disease. This occurred 4 months after the initiation of treatment (15). Pahouja et al. showed that a patient with symptomatic total bone marrow failure survived for 44 months with continuous low-dose doxorubicin followed by hormone treatment (16). Kopp et al. reported that 22 cases with symptomatic bone marrow metastasis had a median overall survival of 19 months during treatment with a cytotoxic regimen. However, the most common complications of bone marrow metastasis were anemia and thrombocytopenia. No patients with total marrow failure were found (8). As expected not all patients responded to treatment.

In our case, the symptoms of fatigue and anemia were seen because of bone marrow metastasis and pancytopenia. Kopp et $a l$. pointed out that the best response rate ( 5 of 22 cases) was documented after treatment with docetaxel/adriamycin (8). However, we chose multidrug therapy including anthracycline drugs, doxorubicin and cyclophosphamide treatment, which is a typical standard chemotherapy for breast cancer (17). She was able to achieve sustained disease control through doxorubicin and cyclophosphamide followed by docetaxel, without bleeding complications at the first recurrence of bone marrow metastasis. In addition, no evidence of disseminated intravascular coagulation was observed in our case.

At 15 months after doxorubicin and cyclophosphamide was completed, a second relapse of bone marrow metastasis occurred. We decided on eribulin after thinking about the treatment strategy for recurrent bone marrow metastasis. Eribulin is a non-taxane inhibitor of microtubule dynamics, which belongs to the halichondrin class of anti-neoplastic agents (18). In addition to its primary function, preclinical studies have suggested other potential benefits of these drugs, such as preventing epithelial-mesenchymal transition to inhibit cancer stem cell traits and improving the tumor 


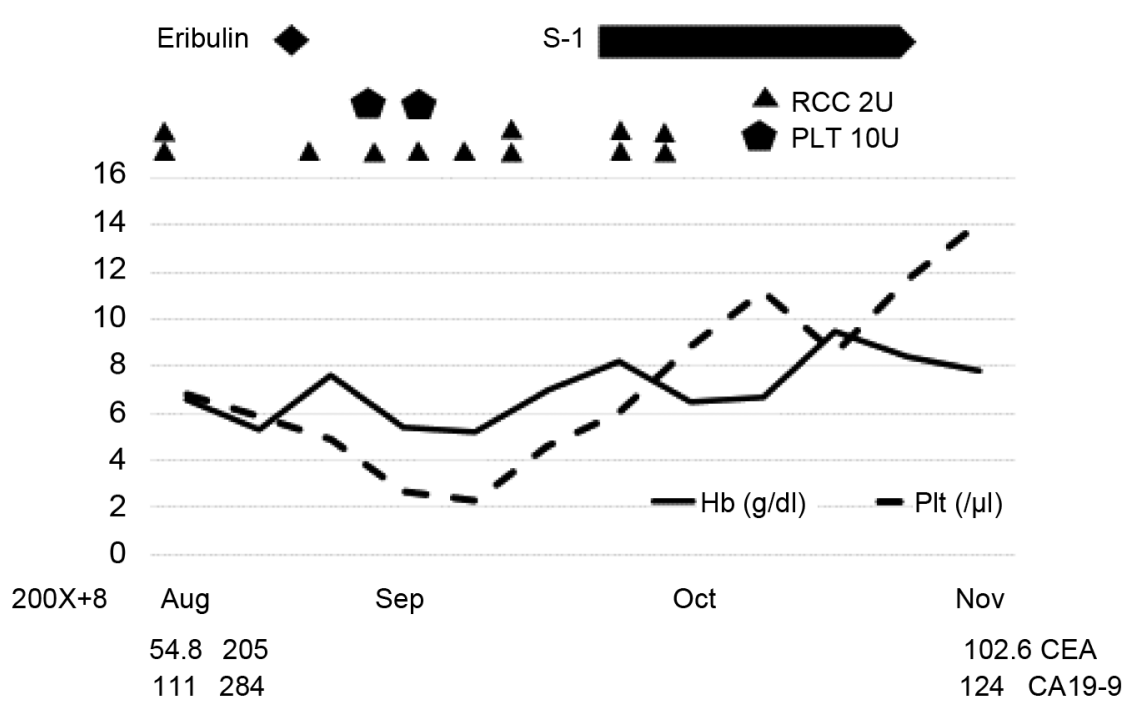

Figure 3. At the time of the second bone marrow metastasis, both $\mathrm{Hb}$ and platelet counts were quite low, suggesting that eribulin was at great risk. However, with just one cycle of eribulin, both $\mathrm{Hb}$ and platelet levels stabilized after overcoming myelosuppression. She survived her second bone marrow metastasis of breast cancer.

microenviroment by vascular remodeling to reduce drug resistance and metastasis (19-21). The 305/EMBRACE study has revealed that eribulin as third- or later-line chemotherapy for metastatic breast cancer improved overall survival in comparison to the treatments selected by physicians (22). Accordingly, eribulin was administered at the second relapse of bone marrow metastasis. As Figure 3 demonstrates, she survived this second instance of bone marrow metastasis with just one administration of eribulin. We did not perform bone marrow aspiration or biopsy; our judgments were based on the results of her blood tests.

Kopp et al. reported that the partial response rate and the stable disease rate were $27 \%$ and $28 \%$, respectively, as the best response (8). In our case, a partial response was observed after the first recurrence, while stable disease was maintained after the second recurrence. According to Kopp's study, the median survival time from the date of the diagnosis of bone marrow metastasis was 19 months. It was very fortunate that she survived for 38 months after the first diagnosis of bone marrow metastasis.

\section{Conflicts of Interest}

The Authors have no conflicts of interest to declare regarding this study.

\section{Authors' Contributions}

Conception and design: Hideko Akagi, Akihiko Shimada. Analysis and interpretation: Hideko Akagi, Akihiko Shimada, Kenko Chin, Hideharu Domoto. Data collection: Hideko Akagi, Akihiko Shimada,
Kenko Chin, Hideharu Domoto. Writing the article: Hideko Akagi. Critical revision of the article: Akihiko Shimada, Kenko Chin, Hideharu Domoto. Final approval of the article: Hideko Akagi, Akihiko Shimada, Kenko Chin, Hideharu Domoto.Overall responsibility: Hideko Akagi.

\section{References}

1 Fahad Ullah M: Breast cancer: current perspectives on the disease status. Adv Exp Med Biol 1152: 51-64, 2019. PMID: 31456179. DOI: 10.1007/978-3-030-20301-6_4

2 Kennecke H, Yerushalmi R, Woods R, Cheang MC, Voduc D, Speers CH, Nielsen TO and Gelmon K: Metastatic behavior of breast cancer subtypes. J Clin Oncol 28(20): 3271-3277, 2010. PMID: 20498394. DOI: 10.1200/JCO.2009.25.9820

3 Early Breast Cancer Trialists' Collaborative Group (EBCTCG): Effects of chemotherapy and hormonal therapy for early breast cancer on recurrence and 15-year survival: an overview of the randomised trials. Lancet 365(9472): 1687-1717, 2005. PMID: 15894097. DOI: 10.1016/S0140-6736(05)66544-0

4 Manders K, van de Poll-Franse LV, Creemers GJ, Vreugdenhil G, van der Sangen MJ, Nieuwenhuijzen GA, Roumen RM and Voogd AC: Clinical management of women with metastatic breast cancer: a descriptive study according to age group. BMC Cancer 6: 179, 2006. PMID: 16824210. DOI: 10.1186/1471-2407-6-179

5 Ahn SG, Lee HM, Cho SH, Lee SA, Hwang SH, Jeong J and Lee HD: Prognostic factors for patients with bone-only metastasis in breast cancer. Yonsei Med J 54(5): 1168-1177, 2013. PMID: 23918566. DOI: 10.3349/ymj.2013.54.5.1168

6 Kamby C, Guldhammer B, Vejborg I, Rossing N, Dirksen H, Daugaard S and Mouridsen HT: The presence of tumor cells in bone marrow at the time of first recurrence of breast cancer. Cancer 60(6): 1306-1312, 1987. PMID: 3621113. DOI: 10.1002/10970142(19870915)60:6<1306::aid-cncr2820600624>3.0.co;2-x 
7 Klevesath MB, Pantel K, Agbaje O, Provenzano E, Wishart GC, Gough P, Pinder SE, Duffy S and Purushotham AD: Patterns of metastatic spread in early breast cancer. Breast 22(4): 449-454, 2013. PMID: 23726130. DOI: 10.1016/j.breast.2013.04.017

8 Kopp HG, Krauss K, Fehm T, Staebler A, Zahm J, Vogel W, Kanz L and Mayer F: Symptomatic bone marrow involvement in breast cancer-clinical presentation, treatment, and prognosis: a single institution review of 22 cases. Anticancer Res 31(11): 4025-4030, 2011. PMID: 22110237.

9 Iguchi H: Recent aspects for disseminated carcinomatosis of the bone marrow associated with gastric cancer: What has been done for the past, and what will be needed in future? World J Gastroenterol 21(43): 12249-12260, 2015. PMID: 26604634. DOI: $10.3748 /$ wjg.v21.i43.12249

10 Rieker O, Grünwald F, Layer G, Kurbacher CM, Hotze AL, Menzel $\mathrm{C}$ and Biersack $\mathrm{HJ}$ : Disseminated bone marrow metastases from primary breast cancer: detection and follow-up by radioimmune bone marrow scintigraphy. J Nucl Med 35(9): 1485-1487, 1994. PMID: 8071697.

11 Kanno M, Nakamura S, Uotani C, Yamanaka S, Terasaki Y, Tsugawa K and Noguchi M: Prognosis of node-positive breast cancer patients who underwent parasternal lymph node biopsy during surgery followed by doxorubicin- or mitoxantronecontaining adjuvant chemotherapy. J Chemother 12(5): 435-441, 2000. PMID: 11128565. DOI: 10.1179/joc.2000.12.5.435

12 Ishikawa T, Shimizu D, Kito A, Ota I, Sasaki T, Tanabe M, Yamada A, Arioka H, Shimizu S, Wakasugi J, Mori R, Chishima $\mathrm{T}$, Ichikawa $\mathrm{Y}$ and Endo I: Breast cancer manifested by hematologic disorders. J Thorac Dis 4(6): 650-654, 2012. PMID: 23205295. DOI: 10.3978/j.issn.2072-1439.2012.10.17

13 Takayama S, Ueno H, Kawaguchi S, Asahara F, Hoshimoto S, Harada H, Ogawa S, Sato M, Matsui J and Ando N: [A case of bone marrow carcinomatosis from breast cancer treated with weekly Paclitaxel]. Gan To Kagaku Ryoho 39(1): 99-101, 2012. PMID: 22241360.

14 Kawai H, Sugimoto R, Miyauchi S, Yoshida R, Waki N, Hirayama S, Ishizaki M, Nishi $\mathrm{H}$ and Yamashita K: [A case of bone marrow carcinomatosis with disseminated intravascular coagulation arising from breast cancer successfully treated with paclitaxel plus bevacizumab]. Gan To Kagaku Ryoho 41(12): 1957-1959, 2014. PMID: 25731388.

15 Sasada S, Yokoyama G, Takahashi H, Koike K, Ikejiri K and Fujii T: [A case of recurrent breast cancer with bone marrow metastasis treated with weekly paclitaxel therapy]. Gan To Kagaku Ryoho 35(9): 1611-1613, 2008. PMID: 18799923.

16 Pahouja G, Wesolowski R, Reinbolt R, Tozbikian G, Berger M, Mangini N and Lustberg MB: Stabilization of bone marrow infiltration by metastatic breast cancer with continuous doxorubicin. Cancer Treat Commun 3: 28-32, 2015. PMID: 25914871. DOI: $10.1016 /$ j.ctrc.2014.11.002
17 Andreopoulou E and Sparano JA: Chemotherapy in patients with anthracycline- and taxane-pretreated metastatic breast cancer: an overview. Curr Breast Cancer Rep 5(1): 42-50, 2013. PMID: 23440080. DOI: 10.1007/s12609-012-0097-1

18 Huyck TK, Gradishar W, Manuguid F and Kirkpatrick P: Eribulin mesylate. Nat Rev Drug Discov 10(3): 173-174, 2011. PMID: 21358731. DOI: $10.1038 / \mathrm{nrd} 3389$

19 Yoshida T, Ozawa Y, Kimura T, Sato Y, Kuznetsov G, Xu S, Uesugi M, Agoulnik S, Taylor N, Funahashi Y and Matsui J: Eribulin mesilate suppresses experimental metastasis of breast cancer cells by reversing phenotype from epithelialmesenchymal transition (EMT) to mesenchymal-epithelial transition (MET) states. Br J Cancer 110(6): 1497-1505, 2014. PMID: 24569463. DOI: 10.1038/bjc.2014.80

20 Funahashi Y, Okamoto K, Adachi Y, Semba T, Uesugi M, Ozawa Y, Tohyama O, Uehara T, Kimura T, Watanabe H, Asano M, Kawano S, Tizon X, McCracken PJ, Matsui J, Aoshima K, Nomoto $\mathrm{K}$ and Oda $\mathrm{Y}$ : Eribulin mesylate reduces tumor microenvironment abnormality by vascular remodeling in preclinical human breast cancer models. Cancer Sci 105(10): 1334-1342, 2014. PMID: 25060424. DOI: 10.1111/cas.12488

21 Mani SA, Guo W, Liao MJ, Eaton EN, Ayyanan A, Zhou AY, Brooks M, Reinhard F, Zhang CC, Shipitsin M, Campbell LL, Polyak K, Brisken C, Yang J and Weinberg RA: The epithelialmesenchymal transition generates cells with properties of stem cells. Cell 133(4): 704-715, 2008. PMID: 18485877. DOI: 10.1016/j.cell.2008.03.027

22 Cortes J, O'Shaughnessy J, Loesch D, Blum JL, Vahdat LT, Petrakova K, Chollet P, Manikas A, Diéras V, Delozier T, Vladimirov V, Cardoso F, Koh H, Bougnoux P, Dutcus CE, Seegobin S, Mir D, Meneses N, Wanders J, Twelves C and EMBRACE (Eisai Metastatic Breast Cancer Study Assessing Physician's Choice Versus E7389) investigators: Eribulin monotherapy versus treatment of physician's choice in patients with metastatic breast cancer (EMBRACE): a phase 3 open-label randomised study. Lancet 377(9769): 914-923, 2011. PMID: 21376385. DOI: 10.1016/S0140-6736(11)60070-6 